\title{
Multidisciplinary treatment of chronic active Epstein-Barr virus infection with multiple complications: a case report
}

\author{
Ahmed Faisal Ali ${ }^{1}$, Juanjuan Liu ${ }^{1}$, Shiqiang Shang ${ }^{2}$, Yinghu Chen ${ }^{1}$ \\ ${ }^{1}$ Department of Infectious Diseases, The Children's Hospital, Zhejiang University School of Medicine, Hangzhou, China; ${ }^{2}$ Department of Clinical \\ Laboratory, The Children's Hospital, Zhejiang University School of Medicine, Hangzhou, China \\ Correspondence to: Shiqiang Shang, MD, PhD. Department of Clinical Laboratory, The Children's Hospital, Zhejiang University School of Medicine, \\ 3333 Bincheng Road, Hangzhou 310052, China. Email: shangsq@zju.edu.cn; Yinghu Chen. Department of Infectious Diseases, The Children’s \\ Hospital, Zhejiang University School of Medicine, 3333 Bincheng Road, Hangzhou 310052, China. Email: cyh18@zju.edu.cn.
}

\begin{abstract}
Chronic active Epstein-Barr virus (CAEBV) infection is a very rare and potentially lifethreatening illness caused by long-term EBV infection. Globally, the prevalence is the highest among young children and adolescents with increased frequency in Asians and Native Americans, whereas it is sporadically encountered in European countries. Typically, patients present with nonspecific symptoms such as fever, lymphadenopathy, hepatosplenomegaly, and liver dysfunction. However, the complications of CAEBV and its treatment are quite complex and require great care. We report a case of a 3-year-old boy with CAEBV infection who was later diagnosed with pancreatic mass and recurrent pancreatitis. A multidisciplinary board was consulted for correct diagnosis and treatment plan making. The treatment included pharmaceutical and surgical (duodenum-preserving pancreatic head resection) approaches. The patient showed tremendous improvement following the third discharge from the hospital and is still free of any symptoms. In this case report, we discuss differential diagnosis and comprehensively examined the possibility of the development of pancreatitis caused by EBV infection, the possibility of autoimmune pancreatitis, and the possibility of hereditary pancreatitis. To confirm or rule out the first two etiologies, laboratory and pathology results were studied. We also performed exon sequencing using Agilent exome capture kit to rule out hereditary pancreatitis. The clinical course of this disease and the way it was handled deserves attention so that similar cases receive prompt treatment.
\end{abstract}

Keywords: Epstein-Barr virus infection (EBV infection); chronic active Epstein-Barr virus infection (CAEBV infection); pancreatitis; case report

Submitted Apr 07, 2021. Accepted for publication Jun 17, 2021.

doi: $10.21037 /$ tp-21-154

View this article at: https://dx.doi.org/10.21037/tp-21-154

\section{Introduction}

Chronic active Epstein-Barr virus (CAEBV) infection is a very rare and potentially life-threatening illness caused by long-term EBV infection. Globally, the prevalence is the highest among young children and adolescents with increased frequency in Asians and Native Americans, whereas it is sporadically encountered in European countries. Typically, these patients have unspecific symptoms such as fever, lymphadenopathy, hepatosplenomegaly, and liver dysfunction (1).
We report a case of a 3-year-old boy diagnosed with CAEBV infection followed by pancreatic mass and recurrent pancreatitis. Due to the difficult clinical course of this patient's illness and complex diagnosis making, specialists from various departments were consulted. The multidisciplinary approach was crucial for the correct identification of the etiology of pancreatitis and pancreatic mass and selecting the most suitable treatment option. We present the following case in accordance with the CARE reporting checklist (available at https://dx.doi.org/10.21037/ tp-21-154). 
Table 1 Results of EBV DNA test

\begin{tabular}{lcc}
\hline Date & Specimen & Viral load $($ copy $/ \mathrm{mL})$ \\
\hline July 14 & Blood serum & $1.45 \times 10^{5}$ \\
July 19 & Blood serum & $8.94 \times 10^{4}$ \\
July 21 & CSF & Negative \\
July 21 & Bone marrow & $2.57 \times 10^{5}$ \\
July 24 & Blood serum & $2.40 \times 10^{4}$ \\
July 31 & Blood serum & $3.17 \times 10^{4}$ \\
August 3 & Blood serum & $1.8 \times 10^{4}$ \\
\hline
\end{tabular}

\section{Case presentation}

A 3-year-old Chinese boy was admitted to our hospital with fever, decreased appetite, and dry cough. He was diagnosed with upper respiratory disease in the local hospital and treated with antibiotics without any sign of improvement. His past medical and family histories were unremarkable. The physical examination revealed throat congestion, mild tonsillitis, cervical lymphadenopathy, hepatosplenomegaly, and skin rash mostly on the face. No abnormalities were found on chest auscultation.

Laboratory findings on admission included a decreased lymphocyte count of $2.71 \times 10^{9} / \mathrm{L}$ (lymphocytes: $61 \%$, neutrophils: $28 \%$ ), hemoglobin of $101 \mathrm{~g} / \mathrm{L}$, CRP of $6 \mathrm{mg} / \mathrm{L}$, ALT of $116 \mathrm{U} / \mathrm{L}$, AST of $127 \mathrm{U} / \mathrm{L}$, and LDH of 1,174 U/L. EBV was detected in different specimens (blood serum and bone marrow) using DNA analysis (Table 1). EBV antibody test results were as follows: EBNA-IgG positive (2.67), EB VCA-IgG positive (108.67 U/mL), EBV-VCA IgM negative. Chest X-ray, ECG, and echocardiography did not show any abnormalities. MRI scan of the head showed abnormal plaques in the deep white matter of the left frontal lobe. Ultrasonography supported physical examination findings: hepatomegaly, splenomegaly, enlarged splenic hilum lymph nodes, and bilateral cervical lymph nodes (right: $2.5 \mathrm{~cm}$ $\times 1.3 \mathrm{~cm}$, left: $1.4 \mathrm{~cm} \times 0.8 \mathrm{~cm}$ ).

The final diagnosis included EBV infection with viral encephalitis and bone marrow involvement was made. The patient received IVIG (10 g/dose, qd, 2 days), ganciclovir with glycyrrhizin, and methylprednisolone $(1 \mathrm{~g} / \mathrm{kg}$, qd, 2 days). AST and ALT normalized after 12 days. The patient was then discharged from the hospital with prednisone.

About 1.5 months later, he was readmitted due to fever and abdominal pain. Apart from high serum EBV DNA level $\left(4.97 \times 10^{4}\right.$ copies/mL), only CRP and ALT were elevated $(17 \mathrm{mg} / \mathrm{L}$ and $52 \mathrm{U} / \mathrm{L}$, respectively). Abdominal ultrasound detected a hypoechoic mass $(2.5 \mathrm{~cm} \times 2 \mathrm{~cm}$ $\times 2.3 \mathrm{~cm})$ in the head of the pancreas, pancreatic head compression, and cervical lymphadenopathy. MRCP scan located the mass in the uncinate process (Figure 1).

Based on these results, we consulted pediatric surgeons that decided to proceed with duodenum-preserving pancreatic head resection (end-to-end choledochojejunostomy and Roux-Y pancreatojejunostomy). Both pancreatic tissue and lymph nodes were harvested for pathology (Figure 2). The pathological report stated pancreatitis (EBER negative) and reactive hyperplasia of lymph nodes (several lymph nodes were EBER positive), IgG4 negative. The common bile duct was thickened with inflammatory cell infiltration and tissue fibrosis. One week after surgery, pancreatic pseudocysts were discovered on MRI.

The patient consecutively received roxifen $0.9 \mathrm{~g} \mathrm{qd}$, sulperazone $0.85 \mathrm{~g}$ bid, and sulperazone $160 \mathrm{mg}$ qh. After the abdominal pain was alleviated and the laboratory results normalized, the patient was discharged from the hospital.

After 6 months, the patient came again with chief complaints of abdominal pain and vomiting ( $>10$ times a day). His vitals were normal, and general physical examination did not reveal any abnormalities. Laboratory findings included liver enzymes and CBC within normal range, CRP $41 \mathrm{mg} / \mathrm{L}$, serum amylase $924.5 \mathrm{U} / \mathrm{L}$, serum lipase 2,244.8 U/L urinary amylase 13,780 U/ L, serum EBV DNA negative, antinuclear and antineutrophil antibodies negative. Ultrasonography showed enlarged pancreas and proximal common bile duct slight dilatation. MRCP indicated an absence of pancreatic head with enlarged pancreatic body and tail. Based on ultrasonography and MRCP results, the final diagnosis of recurrent pancreatitis and postoperative cystic lesion at the pancreatic head was made. The treatment was largely symptomatic and consisted of fasting, rehydration, etc. Abdominal pain and vomiting quickly resolved, and 1 week later the patient resumed an ordinary diet. The patient showed tremendous improvement following the third discharge and is still free of any symptoms.

Agilent exome capture kit used to sequence exons produced paired-end sequencing data with an average depth of $>100 \times$. Each read was $2 \times 150 \mathrm{bp}$, and thus 11.9 million read pairs were generated. We included: (I) 6 genes most frequently found in association with acute pancreatitis and hereditary pancreatitis; (II) 84 genes related to acute pancreatitis and hereditary pancreatitis found on 

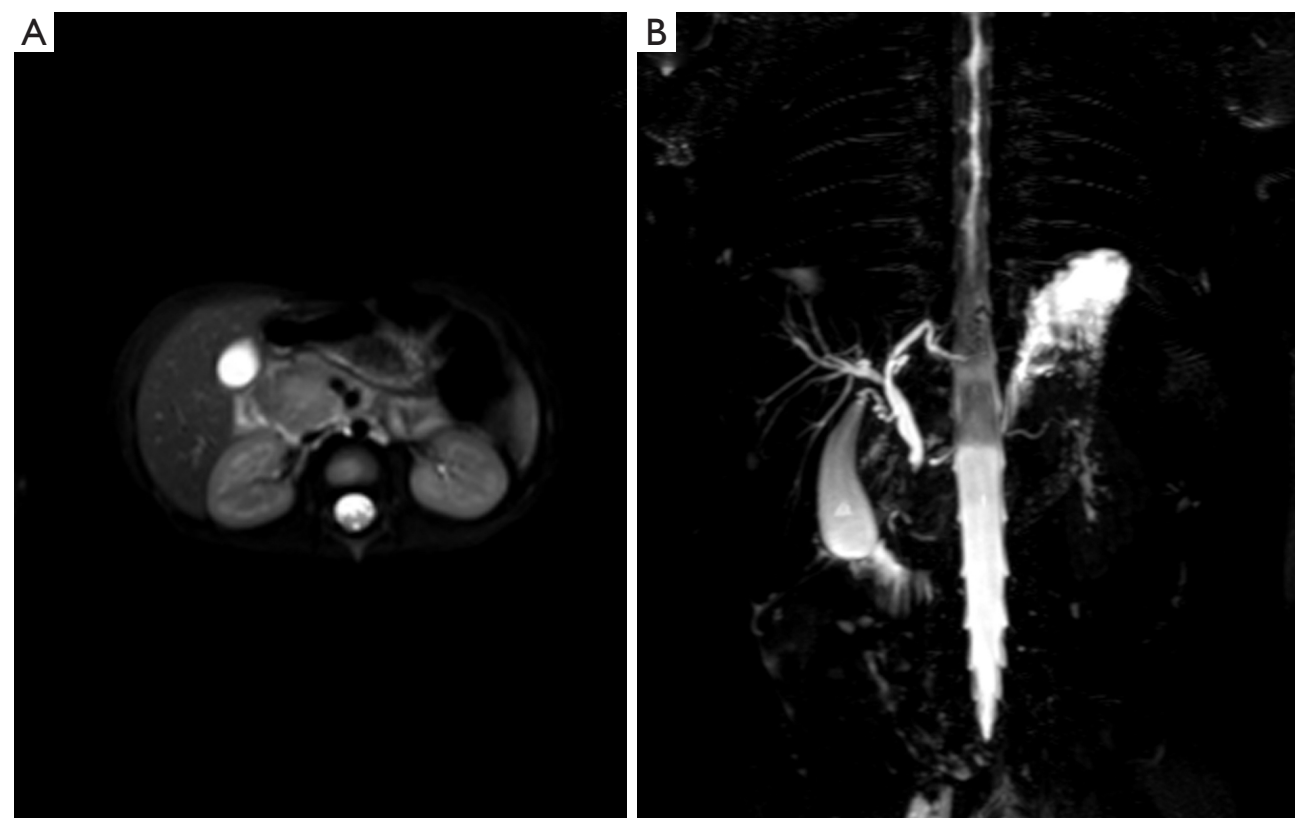

Figure 1 MRCP scan showing the lesion in the uncinate process of the pancreas (A) mild dilatation of the bile duct and the pancreatic duct (B).
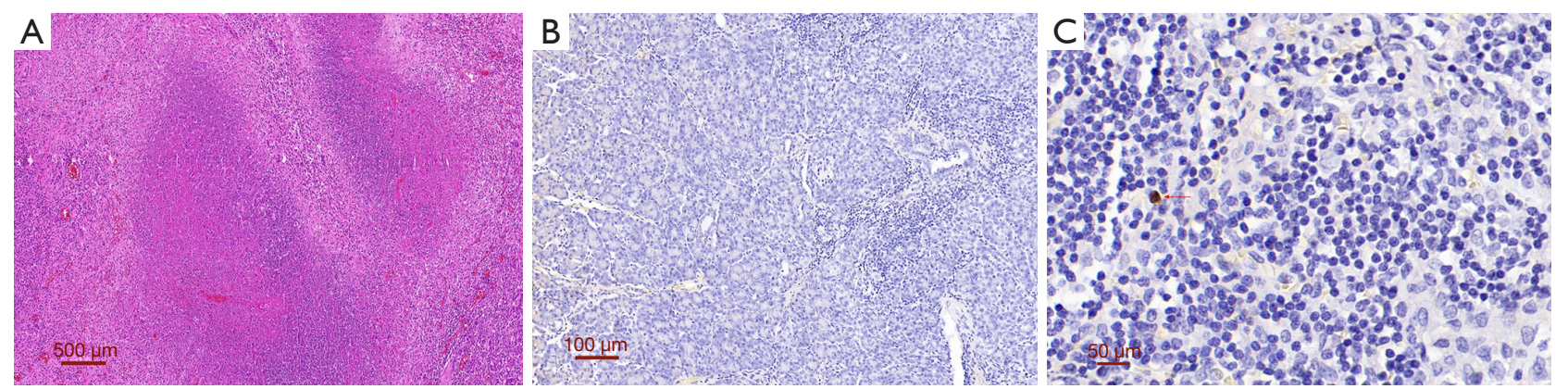

Figure 2 Pathology results. First routine H\&E staining was performed, then Envision ${ }^{\mathrm{TM}}$ double stain was applied for staining CD68, AFP, and IgG4, after which EBER in situ hybridization stain was used. (A) Pancreatic necrosis (50×); (B) pancreatic EBER negative (100×); (C) some EBER positive cells (arrow) of peripheral lymph nodes (400×).

PubMed and relevant databases with independent datamining algorithm; (III) 210 immune-related genes with high reference value sorted by Invitae and other testing companies; (IV) 3,874 genes from OMIM database; (V) 59 key clinically common genes recommended by American Genetic Society. No clear connection between CAEBV and recurrent pancreatitis was established. The genetic cause of the disease is unknown.

All procedures performed in studies involving human participants were in accordance with the ethical standards of the institutional and/or national research committee(s) and with the Helsinki Declaration (as revised in 2013). Written informed consent was obtained from the patient.

\section{Discussion}

The clinical manifestations of our patient on the first admission included fever, symptoms of upper airway infection, and cervical lymphadenopathy that correlate with nonspecific EBV infection (1).

CAEBV alters CD8 cell function making it impossible to clear B cells infected by EBV, leading to a chronic active infection (2). EBV can affect not only B cells but also $\mathrm{T}$ cells and NK cells and may induce lymphoma and 
lymphoproliferative diseases (3). The patient initially suffered from EBV infection, but whether it was CAEBV remains controversial because of its poor prognosis whereas the child's condition was generally good. Nonetheless, most of our experts agree with the diagnosis of CAEBV. Moreover, this patient's EBV-DNA copy number was highest in T cells after turning negative in serum, which suggests that $\mathrm{T}$ cells were more likely to be infected by EBV during the active phase of CAEBV.

$\mathrm{EBV}$ infection is known to lead to pancreatitis. However, postoperative pathology of pancreas showed EBER-negative cells, which makes a direct connection between EBV and pancreatitis inconclusive and supportive of autoimmune pancreatitis (AIP). But the diagnosis of AIP cannot be made because of normal serum IgG4 level and negative IgG4 on pathology (4). Thus, AIP type I can be excluded. Besides, it usually occurs in older men rather than children. Patients with AIP type II can have local tumor-like lesions with generally homogenous pancreatic lesions characterized by plasma cell/neutrophils/pancreatic duct epithelial cells infiltration (5). In our case, the middle part of the pancreatic head tumor was hypoechoic, and the pathology result was not coherent with AIP type II as no infiltration of neutrophils in the pancreatic duct and no damage to ductal epithelium were found.

Pancreatic surgery was advised because of the presence of symptoms such as abdominal pain, elevated pancreatic lipase, and dilatation of both common bile and pancreatic ducts. Surgery excludes any possible malignancies. The postoperative pathology report did not reveal any tumor cells. It was suggestive of possible chronic pancreatitis due to pancreatic tissue fibrosis. Chronic pancreatitis in children is often caused by congenital anatomic abnormalities. However, MRCP did not reveal the presence of any pancreatic or biliary tree anomalies. Recurrent pancreatitis was immediately suspected on the third admission because of characteristic presentation: abdominal pain, vomiting, significantly elevated blood amylase and lipase, and pancreatic tail exudation on ultrasound. Hereditary pancreatitis is linked to mutations in PRSS1, CFTR, SPINK1, CTSB, CASR, PSP/Reg, etc. (6-9). But no genes associated with hereditary pancreatitis were found.

There are many explanations of the connection between EBV infection and pancreatitis. Firstly, it may be a direct invasion of EBV, but no evidence was found on pathological examination. Secondly, there is a possibility of EBV invasion of the liver and subsequent immune changes that lead to pancreatitis. It is theoretically possible but cannot explain the second pancreatitis onset. Also, specifics of the surgery can rule out the biliary etiology of pancreatitis. Thirdly, EBV infection may induce the release of cytokines and inflammatory mediators, causing inflammation of the pancreas. Finally, pancreatitis could be attributed to genetic causes, but the existence of related gene mutations is unclear.

\section{Conclusions}

Long-term follow-up is necessary in this case. In addition, further validation of parental genotyping and further analysis of the immune status of the child by more detailed lymphocyte-subset function tests (including T-lymphocyte proliferation tests) are needed. If pancreatitis recurs, a pancreatic stent needs to be placed. The patient's parents were content with the treatment and said that "their baby went back to normal status".

\section{Acknowledgments}

We would like to thank each member of the multidisciplinary team that worked hard in the treatment of this patient. Finally, we would like to express our profound gratitude to Bulat Abdrakhimov for his help and valuable comments he provided during writing and publishing this article as well as language editing.

Funding: This study was supported by the National Science Foundation of China (82071812), the National Science Foundation of Zhejiang Province, China (LY19H00003).

\section{Footnote}

Reporting Checklist: The authors have completed the CARE reporting checklist. Available at https://dx.doi. org/10.21037/tp-21-154

Peer Review File: Available at https://dx.doi.org/10.21037/ tp-21-154

Conflicts of Interest: All authors have completed the ICMJE uniform disclosure form (available at https://dx.doi. org/10.21037/tp-21-154). The authors have no conflicts of interest to declare.

Ethical Statement: The authors are accountable for all aspects of the work in ensuring that questions related to the accuracy or integrity of any part of the work are 
appropriately investigated and resolved. All procedures performed in studies involving human participants were in accordance with the ethical standards of the institutional and/or national research committee(s) and with the Helsinki Declaration (as revised in 2013). Written informed consent was obtained from the patient.

Open Access Statement: This is an Open Access article distributed in accordance with the Creative Commons Attribution-NonCommercial-NoDerivs 4.0 International License (CC BY-NC-ND 4.0), which permits the noncommercial replication and distribution of the article with the strict proviso that no changes or edits are made and the original work is properly cited (including links to both the formal publication through the relevant DOI and the license). See: https://creativecommons.org/licenses/by-nc-nd/4.0/.

\section{References}

1. Wass M, Bauer M, Pfannes R, et al. Chronic active Epstein-Barr virus infection of T-cell type, systemic form in an African migrant: case report and review of the literature on diagnostics standards and therapeutic options. BMC Cancer 2018;18:941.

2. Ai J, Xie Z. Epstein-Barr Virus-Positive T/NK-Cell Lymphoproliferative Diseases in Chinese Mainland. Front

Cite this article as: Ali AF, Liu J, Shang S, Chen Y. Multidisciplinary treatment of chronic active Epstein-Barr virus infection with multiple complications: a case report. Transl Pediatr 2021;10(9):2402-2406. doi: 10.21037/tp-21-154
Pediatr 2018;6:289.

3. Dojcinov SD, Fend F, Quintanilla-Martinez L. EBVPositive Lymphoproliferations of B- T- and NKCell Derivation in Non-Immunocompromised Hosts. Pathogens 2018;7:28.

4. Okazaki K, Uchida K. Current perspectives on autoimmune pancreatitis and IgG4-related disease. Proc Jpn Acad Ser B Phys Biol Sci 2018;94:412-27.

5. Ito T, Kawa S, Matsumoto A, et al. Risk Factors for Pancreatic Stone Formation in Type 1 Autoimmune Pancreatitis: A Long-term Japanese Multicenter Analysis of 624 Patients. Pancreas 2019;48:49-54.

6. Shelton CA, Umapathy C, Stello K, et al. Hereditary Pancreatitis in the United States: Survival and Rates of Pancreatic Cancer. Am J Gastroenterol 2018;113:1376.

7. Bellin MD, Prokhoda P, Hodges JS, et al. Age and Disease Duration Impact Outcomes of Total Pancreatectomy and Islet Autotransplant for PRSS1 Hereditary Pancreatitis. Pancreas 2018;47:466-70.

8. Patel J, Madan A, Gammon A, et al. Rare hereditary cause of chronic pancreatitis in a young male: SPINK1 mutation. Pan Afr Med J 2017;28:110.

9. Avanthi US, Bale G, Aslam M, et al. PRSS1 (R122H) mutation in an Indian family with low penetrance is associated with pancreatitis phenotype. Indian J Gastroenterol 2018;37:67-9. 